\title{
An Effective Prediction of Biomagnification Factors for Organochlorine Pollutants
}

\author{
Ming Cai Zhang \\ Lanzhou University \\ Ke Xin Bi \\ Lanzhou University \\ Bin Qiang Zhao \\ Lanzhou University \\ Hai Ping Shao \\ Lanzhou University
}

Hong Lin Zhai ( $\nabla$ zhaihl@163.com )

Lanzhou University https://orcid.org/0000-0001-7088-4962

\section{Research Article}

Keywords: Biomagnification factors (BMF), Organochlorine pollutants, molecular structure, Tchebichef image moment (TM), Quantitative structure-property relationship (QSPR)

Posted Date: February 19th, 2021

DOI: https://doi.org/10.21203/rs.3.rs-243149/v1

License: (c) (i) This work is licensed under a Creative Commons Attribution 4.0 International License.

Read Full License 


\section{An effective prediction of biomagnification factors for organochlorine pollutants}

Ming Cai Zhang, Hong Lin Zhai ${ }^{*}$, Ke Xin Bi, Bin Qiang Zhao, Hai Ping Shao

College of Chemistry \& Chemical Engineering, Lanzhou University, Lanzhou, 730000, PR China

\section{Abstract}

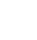

Biomagnification factor (BMF) is an important index of pollutants in food chains but its experimental determination is quite tedious. In this contribution, as the feature descriptors of molecular information, Tchebichef moments (TMs) were calculated from their structural images.

Then stepwise regression was employed to establish the prediction model for the $\log B M F$ of organochlorine pollutants. The correlation coefficient with leave-one-out cross-validation $\left(R_{c v}\right)$ was 0.9570 and the correlation coefficient of prediction $\left(R_{p}\right)$ for external independent test set was 0.9594 . Compared with traditional two-dimensional (2D) quantitative structure-property relationship (QSPR) and the reported augmented multivariate image analysis applied to QSPR (aug-MIA-QSPR), the proposed approach is more simple, accurate and reliable. This study not only obtained the model with better stability and predictive ability for the BMF of organochlorine pollutants, but also provided another effective approach to QSPR research.

Keywords: Biomagnification factors (BMF); Organochlorine pollutants; molecular structure; Tchebichef image moment (TM); Quantitative structure-property relationship (QSPR)

\footnotetext{
* Correspondence to: Tel.: +86 931 8912596; fax: +86 931 8912582; E-mail address: zhaihl@163.com (H.L. Zhai).
} 


\section{Introduction}

With the developments of modern industries, the problem of environmental pollution has been more and more payed attention because that influences human health. Persistent organic pollutions (POPs) are one of the main factors contributing to environmental pollutions, which include three categories: certain industrial chemicals, certain by-products and contaminants, and certain industrial processes (Hillman 1998). A great many POPs are organochlorine compounds (Rosa Vilanova 2001) that easily circulate into organisms with ecosystem cycles (both on land and in aquatic environments), which causes damage to the physiological function of the organisms (Jepson et al. 2016, Paul D. Jepson 2009). More important, since the increasing rate of their concentration is higher than that of degradation due to their stability, organochlorine pollutants can be accumulated along the food chains, which causes a toxicity magnification in the organisms at the top of the food chains (also called Biomagnification phenomenon) (Birgit M. Braune 1989). According to one research of the Lake Ontario ecosystem, it was observed that the content of polychlorinated biphenyls (PCBs) increased with trophic levels (Nliml 1988). To assess this toxicity of POPs, biomagnification factor (BMF) is defined and calculated by the following formula (D. Mackay 2000):

$$
B M F=\frac{\mathrm{c}_{B}}{c_{A}}
$$

where $\mathrm{c}_{B}$ is the concentration of chemical in the organism and $c_{A}$ is the concentration in the organism's diet.

Although the BMFs can be determined by the experimental approaches (Charles J. Henny 2003, Serrano et al. 2008, Woodburn et al. 2013), there are very cumbersome, time consuming and needs 
very specific facilities. Fortunately, quantitative structure-property relationship (QSPR) is one of useful strategies and has been widely applied in various fields, in which several QSPR methods had been used to the prediction of BMF. After calculation a large number of molecular descriptors, the most significant descriptors related to BMF were selected by genetic algorithm and used to build the predictive model of artificial neural network (Fatemi \&Baher 2009). To overcome the limited experimental data and avoid more animal testing, the BMF of PBDEs was assessed by means of QSAR (Mansouri et al. 2012). Augmented multivariate Image Analysis applied to QSPR (aug-MIAQSPR) approach was reported to predict the BMFs of aromatic organochlorine pollutants (da Mota et al. 2017). Acceptable-by-design QSAR method to predict the dietary BMF of organic chemicals in fish, in which two kinds of variable selection methods including genetic algorithms and reshaped sequential replacement were employed (Grisoni et al. 2019). In our opinion, the extraction and selection of features are the most important and key steps in QSAR/QSPR research.

Image moment firstly proposed by $\mathrm{Hu}(\mathrm{Hu} 1962)$ is one of the description methods for grayscale images. Thereafter, a series of image moments have been developed such as Zernike (Teague 1980), Wavelet (Dinggang Shen 1999), Tchebichef (Ramakrishnan Mukundan 2001) and Krawtchouk (Yap et al. 2003) moments. Although these moments are often used to the de-noising or compression in digital image processing, several image moments have been applied to the feature extraction of target information from chemical spectra and employed to establish the analytical models owing to their powerful multi-resolution as well as good invariance (Zhai et al. 2018). As an excellent member of moment family, Tchebichef image moment (TM, also called Chebyshev moment) possesses the more advantages of feature extraction in the analyses of chemical images. In this study, as the novel descriptors of chemical structures, TMs were calculated directly from 
the gray images of molecular structures of organochlorine compounds, and stepwise regression was employed to establish the linear BMF prediction model. The performance of the obtained model was evaluated comprehensively. Furthermore, the results from our approach were compared with that of other methods.

\section{Data and methods}

\subsection{Data set}

The data set was derived from the literatures (da Mota et al. 2017), which consisted of 30 polychlorinated biphenyls (PCBs) congeners and 10 organochlorine pesticides (DDT, DDE, HCB, TCDF, OCDF, TCDD, H6CDD, H7CDD, OCDD and DDD). It was based on the research of $B M F s$ of osprey eggs and whole fish from Willamette River in western Oregon of USA. Their values of $\log B M F$ are listed in Table 1 as Exp. column. All of 40 samples were randomly divided into training set (30 samples) and test set (10 samples). The training set was used to establish the prediction model, and the test set was employed to evaluate the prediction capability of the obtained model as external independent sample set.

\section{(Table 1)}

2.2 Methods

2.2.1 Images of molecular structures

The two-dimensional (2D) molecular structures of 40 organochlorine compounds were drawn in ChemBioDraw (v12) software with default conditions (Fixed Length: $1.058 \mathrm{~cm}$, Line Width: $0.035 \mathrm{~cm}$, Bond Spacing: 12\% of length, Hash Spacing: $0.095 \mathrm{~cm}$, Font: Times New Roman, Size: 12) and saved as the grayscale BMP format with the size of 303 pixels $\times 258$ pixels under the 
resolution ratio of $96 \mathrm{DPI}$.

\subsubsection{Calculation of Tchebichef image moments}

For a given grayscale image with size of $N \times M$, the TM can be calculated using the following formula (Bayraktar et al. 2007):

$$
\begin{aligned}
& T_{n, m}=\frac{1}{\tilde{\rho}(n, N) \tilde{\rho}(m, M)} \sum_{x=0}^{N-1} \sum_{y=0}^{M-1} \tilde{t}_{n}(x) \tilde{t}_{m}(y) f(x, y) \\
& (n=0,1,2, \ldots N-1, \quad m=0,1,2, \ldots M-1)
\end{aligned}
$$

where $\tilde{t}_{n}(x)$ and $\tilde{t}_{m}(y)$ are the normalized discrete Tchebichef polynomial of degree $n$ and $m$, respectively; $\tilde{\rho}(n, N)$ is the squared-norm of the normalized polynomials and $f(x, y)$ is the image intensity function.

Thus the reconstruction of image by $T_{n, m}$ can be performed:

$$
\hat{f}(x, y)=\sum_{n=0}^{n N} \sum_{m=0}^{m M} T_{n m} \tilde{t}_{n}(x) \tilde{t}_{m}(y)
$$

where $\hat{f}(x, y)$ is the reconstructed image, $n N$ and $m M$ are the maximum orders of $n$ and $m(n=0$ $n N, n N<N-1 ; m=0-m M, m M<M-1)$. The reconstruction error $\varepsilon$ can be calculated:

$$
\varepsilon=\sum_{x=0}^{N-1} \sum_{y=0}^{M-1}|f(x, y)-\hat{f}(x, y)|
$$

\subsubsection{Modeling and evaluation}

Stepwise regression was employed to establish the linear prediction model, in which TMs were regarded as the independent variables and $\log B M F$ was denoted as dependent variable. The performance of obtained model was evaluated by means of various statistical parameters, such as the correlation coefficient $\left(R_{c}\right)$, the adjusted correlation coefficient $\left(R_{a d j}\right)$, root mean square error $\left(R M S E_{c}\right)$, the correlation coefficient with leave-one-out (LOO) cross-validation $\left(R_{c v}\right)$ and LOO root mean square error $\left(R M S E_{c v}\right)$ for training set; $F$-test for model and $t$-test for the regression 
104

105

coefficients; the correlation coefficient of test set $\left(R_{p}\right)$ and root mean square error $\left(R M S E_{p}\right)$ for test set (Gadaleta et al. 2016).

In order to further inspect the robustness of the model, a randomized test was performed on the established model, in which models are established with invariant $X$-matrix and randomized $Y$ matrix (Mitra et al. 2010). To determine the reliable of model, ${ }^{c} R_{P}{ }^{2}$ was adopted by following corrected formula (Todeschini 2010):

$$
{ }^{c} R_{p}^{2}=R \sqrt{\left(R^{2}-R_{r}^{2}\right)}
$$

where $R$ is $R_{c}$ of the model and $R_{r}^{2}$ is the average of $R^{2}$ for the randomized model.

The predictive capability of the model can be validated by external test, and the related parameters ( $k, k^{\prime}, r_{m}{ }^{2}, r_{m}{ }^{2}$ and $\Delta r_{m}{ }^{2}$ ) are defined by follows (Ojha et al. 2011, Roy et al. 2013):

$$
k=\frac{\sum\left(Y_{\text {obs }} \times Y_{\text {pred }}\right)}{\sum\left(Y_{\text {pred }}\right)^{2}}
$$

$$
\begin{aligned}
& k^{\prime}=\frac{\sum\left(Y_{o b s} \times Y_{\text {pred }}\right)}{\sum\left(Y_{o b s}\right)^{2}} \\
& r_{m}^{2}=r^{2}\left(1-\sqrt{r^{2}-r_{0}^{2}}\right)
\end{aligned}
$$

$$
\Delta r_{m}^{2}=\left|r_{m}^{2}-r_{m}^{\prime 2}\right|
$$

Here, $k$ and $k^{\prime}$ is the slope of experimental and predicted values respectively. $Y_{o b s}$ and $Y_{\text {pred }}$ are the observed and predicted values, respectively. $r_{m}{ }^{2}$ and $r_{m}{ }^{2}$ are modified $r^{2} . r^{2}$ and $r_{0}{ }^{2}$ are determined coefficient between the observed and predicted values for the least square linear regression with and without interpret. And $\Delta r_{m}^{2}$ is the absolute of the difference between them.

Meanwhile, it is necessary to discuss the applicability domain (AD) of the established model to study its scope and limitations. In this work, Williams plot was employed to calculate the 
125

applicability domain (AD) of the established model, which presents the relationship between leverage (Hat matrix) and standardized residuals, and Hat matrix could be calculated by the following relation (P. 2007):

$$
H=X\left(X^{T} X\right)^{-1} X^{T}
$$

where $X$ is the matrix composed of descriptors in the established model and $T$ means the transpose matrix. In general, the threshold $H^{*}$ of $H$ is equal to $3 p / n$ ( $n$ is the number of training set sample and $p$ is the established model's variables number plus one), and the standardized residuals are normally accepted within the range \pm 3 (Roy et al. 2015).

2.2.4 Comparison with 2D QSPR as well as the reported method

Traditional 2D QSPR method was also applied to the same data set. The molecular descriptors of 40 samples were calculated by CODESSA (v2.63) after being optimized by HyperChem, and the total of 337 common descriptors were obtained (Supporting information, Table S1). A linear QSPR model was established by stepwise regression based on the training set, and used to the prediction of the test set. The obtained results were compared with that of the proposed method.

The proposed model was also applied to predict the $\log B M F$ of the samples in the five different test sets as same as the reference (da Mota et al. 2017), and the calculated results were compared with that of the method in this reference.

\section{Results and discussions}

\subsection{Characteristic of Tchebichef image moments}

Owing to the excellent description ability with multi-resolution and invariance properties in image processing, Tchebichef image moment (TM) is an important image characteristic based on 
147

the discrete orthogonal polynomials (Ramakrishnan Mukundan 2001). What is more, no numerical approximation is needed during the calculation.

TMs with different moment orders represent different information in image according to Eq. 2, which can decompose the information of molecular structure image (multi-resolution ability). Then the important features $\left(T_{n, m}\right)$ related to the BMF of chemical compounds could be selected by stepwise regression to establish analytical model. Owing to its invariance property in the image operation of shifting, scaling and rotation, TMs have relatively stable calculated values, which means that molecular structures do not need to be precisely aligned in their images.

3.2 Model and evaluation

After the TMs were directly calculated from the grayscale images of molecular structures, the maximum orders were determined as $n N=28$ and $m M=43$ according to the change of reconstruction errors (Eq. 4). Then a linear quantitative model was established by stepwise regression based on the training set, in which the TMs were the independent variables and $\log B M F$ was the target response variable. The TMs of the following model are listed in Table S2.

$$
\begin{aligned}
\log B M F & =-1.0732+92.1940 \times T_{1,1}-6.9062 \times T_{4,14}-10.2245 \times T_{13,9}-5.5451 \times T_{13,12} \\
& +14.7530 \times T_{13,27}
\end{aligned}
$$

The calculated $\log B M F$ values of all samples are listed Table 1 and the statistical parameters of the established model are summarized in Table 2. The linear relationship between the calculated values and the experimental values are shown in Fig. 1 . From Table 2 , the $R_{c}(0.9726), R_{a d j}(0.9668)$ and $R M S E_{c}(0.1052)$ are satisfactory, which indicates that the model is accurate; $R_{c v}(0.9570)$ and $R M S E_{c v}(0.1320)$ suggest that there is not over-fitting; the $p$-value of $F$-test $(2.08 \mathrm{e}-14)$ shows the good linear relationship between the independent variables and response variable in this model, and 
the results of $t$-test ensure that the regression coefficients have statistical significance. For the test set, $R_{p}(0.9594)$ and $R M S E_{p}(0.2129)$ represent that the established model possesses satisfactory predictive ability. All above statistical parameters indicate that the model has high reliability and accuracy.

\section{(Table 2, Fig. 1)}

To investigate the robustness and reliability of the model, the further testing was carried out. The parameter of randomized test $\left({ }^{c} R_{P}^{2}\right)$ is 0.5988 (more than its threshold value of 0.5 ), indicating that the model has not randomness and fortuitousness. For the external test, the obtained parameters (listed in Table 2) also conform to the requirements $\left(0.85 \leq k \leq 1.15 ; 0.85 \leq k^{\prime} \leq 1.15 ; r_{m}{ }^{2} \geq 0.5 ; r_{m}{ }^{2}\right.$ $\geq 0.5 ; \Delta r_{m}^{2} \leq 0.2$ ) (Ojha et al. 2011). Besides, Williams plot is shown Fig. 2A. As it could be observed, sample $\mathbf{8}(1,2,3,4,6,7,8,9$-Octachlorodibenzofuran) of the training set and sample $\mathbf{1}$ (2378TCDF) of test set are outliers with high leverage. Compared with the structures of other chemicals in dataset, sample $\mathbf{8}$ may be different with others so that they are not well modeled by adopted variables. Another possible reason is the sample belongs to other type chemicals. To the sample 1, it owns the same structure with sample $\mathbf{8}$ so that the model may not well predict the value of $\log B M F$ of it.

\section{(Fig. 2)}

All above results and discussions indicate that the proposed method is reliable and reasonable, and the established model possesses robustness and prediction ability.

3.3 Comparison with other methods

\subsubsection{Comparison with 2D-QSPR method}

Based on the 337 common molecular descriptors, the prediction model was established by 
stepwise regression as follows:

$$
\begin{aligned}
\log B M F= & 57.2032+0.0105 \times X_{39}-0.3412 \times X_{162}-499.8468 \times X_{174}+20.8516 \times X_{176} \\
& -42.3347 \times X_{276}-36.4478 \times X_{327}
\end{aligned}
$$

where $X_{39}$, Wiener index; $X_{162}$, No. of occupied electronic levels; $X_{174}$, Avgnucleoph. react. Index for a $\mathrm{Cl}$ atom; $X_{176}$, Max eletroph. react. index for a $\mathrm{C}$ atom; $X_{276}$, Avg bond order of a $\mathrm{Cl}$ atom; $X_{327}$, Principal moment of inertia A.

The calculated values of $\log B M F$ are also listed in Table 1 . The obtained statistical parameters (listed in Table 2) and Williams plot (showed in Fig.2B) illustrate that the established 2D-QSPR model is robust and reliable. The comparison of statistical parameters in the Table 2 indicates that the TM model is slightly better than the 2D-QSPR model the owing to its higher prediction ability, which suggests the feasibility of the proposed approach.

\subsubsection{Comparison with the method in reference}

In the literature (da Mota et al. 2017), the best results were obtained by aug-MIA-QSPR method. By contrast, the TM model had the more satisfied statistical parameters (listed in Table 2). For the five test sets in this literature, the established TM model was also directly used to predict the $\log B M F$ values of the samples, respectively. The obtained statistical parameters $R_{p}{ }^{2}$ and $R M S E_{p}$ are shown in Table 3. It can be seen that the predicted results from the proposed model are

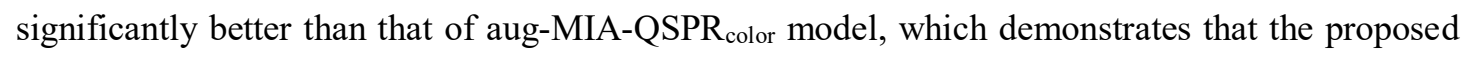
model possesses stronger predictive ability and reliability.

\section{(Table 3)}

\section{Conclusion}



images and establish the linear quantitative model to predict the $\log B M F$ of organochlorine pollutants. The results of comprehensive evaluation indicate that the established model has satisfactory robustness and predictive ability. As an effective extraction pathway of feature information, TM method could be applied on many QSPR research.

\section{Acknowledgement}

This research did not receive any specific grant from funding agencies.

\section{Availability of data and materials}

The datasets used and analyzed during the current study are available from the corresponding author on reasonable request.

\section{Compliance with ethical standards}

Ethics approval and consent to participate Not applicable. 


\section{References}

Bayraktar B, Bernas T, Robinson JP, Rajwa B (2007): A numerical recipe for accurate image reconstruction from discrete orthogonal moments. Pattern Recognition 40, 659-669

Birgit M. Braune RJN (1989): Dynamics of organochlorine compounds in herring gulls: III. Tissue distribution and bioaccumulation in lake ontario gulls. Environmental Toxicology and Chemistry $8,957-968$

Charles J. Henny JLK, Robert A. Grove, V. Raymond Bentley and John E. Elliott (2003): Biomagnification Factors (Fish to Osprey Eggs from Willamette River, Oregon, U.S.A.) for PCDDs, PCDFs, PCBs and OC Pesticides Environmental Monitoring \& Assessment 84, 275 315

D. Mackay AF (2000): Bioaccumulation of persistent organic chemicals: mechanisms and models. Environmental Pollution 110, 375-391

da Mota EG, Duarte MH, Barigye SJ, Ramalho TC, Freitas MP (2017): Exploring MIA-QSPR's for the modeling of biomagnification factors of aromatic organochlorine pollutants. Ecotoxicology and environmental safety $135,130-136$

Dinggang Shen HHSI (1999): Discriminative wavelet shape descriptors for recognition of 2-D patterns. Pattern Recognition 32, 151-165

Fatemi MH, Baher E (2009): A novel quantitative structure-activity relationship model for prediction of biomagnification factor of some organochlorine pollutants. Molecular diversity 13, $343-352$

Gadaleta D, Mangiatordi GF, Catto M, Carotti A, Nicolotti O (2016): Applicability Domain for QSAR Models. International Journal of Quantitative Structure-Property Relationships 1, 45-63

Grisoni F, Consonni V, Vighi M (2019): Acceptable-by-design QSARs to predict the dietary biomagnification of organic chemicals in fish. Integrated Environmental Assessment and Management 15, 51-63

Hillman K (1998): United Nations Economic Commission for Europe: Draft Protocol to the Convention on Long-range Transboundary Air Pollution on Persistent Organic Pollutants*. International Legal Materials 37, 505-529 
Hu M-K (1962): Visual pattern recognition by moment invariants IRE Transactions on Information Theory 8, 179-187

Jepson PD et al. (2016): PCB pollution continues to impact populations of orcas and other dolphins in European waters. Sci Rep 6, 18573

Mansouri K, Consonni V, Durjava MK, Kolar B, Oberg T, Todeschini R (2012): Assessing bioaccumulation of polybrominated diphenyl ethers for aquatic species by QSAR modeling. Chemosphere 89, 433-44

Mitra I, Saha A, Roy K (2010): Exploring quantitative structure-activity relationship studies of antioxidant phenolic compounds obtained from traditional Chinese medicinal plants. Molecular Simulation 36, 1067-1079

Nliml BGOaAJ (1988): Trophodynamic analysis of polychlorinated biphenyl congeners and other chlorinated hydrocarbons in the Lake Ontario ecosystem. Environ Sci Technol 22, 388-397

Ojha PK, Mitra I, Das RN, Roy K (2011): Further exploring rm2 metrics for validation of QSPR models. Chemometrics and Intelligent Laboratory Systems 107, 194-205

P. G (2007): Principles of QSAR models validation: Internal and external. QSAR \& Combinatorial Science 26, 694-701

Paul D. Jepson PMB, Robert Deaville, Colin R. Allchin, John R. Baker, Robin J. Law (2009): Relationships between polychlorinated biphenyls and health status in harbor porpoises (Phocoena phocoena) stranded in the United Kingdom Environmental Toxicology and Chemistry $24,238-248$

Ramakrishnan Mukundan S-HO, P.A. Lee (2001): Image analysis by Tchebichef moments. IEEE Transactions on Image Processing 10, 1357-64

Rosa Vilanova PFn, Carolina Martı'nez, and Joan O. Grimalt (2001): Organochlorine Pollutants in Remote Mountain Lake Waters. Journal of Environmental Quality 30, 1286-1295

Roy K, Chakraborty P, Mitra I, Ojha PK, Kar S, Das RN (2013): Some case studies on application of "r(m)2" metrics for judging quality of quantitative structure-activity relationship predictions: emphasis on scaling of response data. J Comput Chem 34, 1071-82

Roy K, Kar S, Ambure P (2015): On a simple approach for determining applicability domain of QSAR models. Chemometrics and Intelligent Laboratory Systems 145, 22-29 
287 Serrano R, Blanes MA, Lopez FJ (2008): Biomagnification of organochlorine pollutants in farmed and wild gilthead sea bream (Sparus aurata) and stable isotope characterization of the trophic chains. Sci Total Environ 389, 340-9

290 Teague MR (1980): Image analysis via the general theory of moments Journal of the Optical Society of America 70, 920-930

Todeschini R (2010): Milano Chemometrics. University of MilanoBicocca, Milano, Italy (personal communication)

Woodburn K, Drottar K, Domoradzki J, Durham J, McNett D, Jezowski R (2013): Determination of the dietary biomagnification of octamethylcyclotetrasiloxane and decamethylcyclopentasiloxane with the rainbow trout (Oncorhynchus mykiss). Chemosphere 93, 779-88

Yap PT, Paramesran R, Ong SH (2003): Image analysis by Krawtchouk moments. IEEE Trans Image Process 12, 1367-77

Zhai HL, Li BQ, Chen J, Wang X, Xu ML, Liu JJ, Lu SH (2018): Chemical image moments and their applications. TrAC Trends in Analytical Chemistry 103, 119-125

302 
Table Caption:

306 Table 1 Experimental and predicted $\log B M F$ values of all samples

307 Table 2 Performance of the established models

308 Table 3 Comparison of the predicted results for the five test sets

309

310 Figure Caption:

311 Figure 1 Linear relationship between $\log B M F$ calculated values and experimental values

312 Figure 2 Williams plots. (A) TM model $\left(H^{*}=0.6\right)(\mathrm{B}) 2 \mathrm{D}-\mathrm{QSPR}$ model $\left(H^{*}=0.7\right)$ 
Table 1 Experimental and predicted $\log B M F$ values of all samples

\begin{tabular}{|c|c|c|c|c|c|}
\hline \multirow{2}{*}{ No. } & \multirow{2}{*}{ Name } & \multirow{2}{*}{ Notation } & \multicolumn{3}{|c|}{$\log B M F$} \\
\hline & & & Exp. & $T M$ & $2 D-Q S P R$ \\
\hline $1 *$ & 2378TCDF & TCDF & -0.12 & -0.10 & 0.31 \\
\hline 2 & hexachlorobenzene & $\mathrm{HCB}$ & 0.32 & 0.31 & 0.34 \\
\hline 3 & 3,3',4,4'-Tetrachlorobiphenyl & PCB77 & 0.77 & 0.82 & 0.62 \\
\hline 4 & 2,4,4',5-Tetrachlorobiphenyl & PCB74 & 0.83 & 0.89 & 0.96 \\
\hline $5^{*}$ & 2,3,4,4'-Tetrachlorobiphenyl & PCB60 & 0.90 & 0.95 & 0.95 \\
\hline 6 & $2,2^{\prime}, 3,4^{\prime}, 5^{\prime}, 6-$ Hexachlorobiphenyl & PCB149 & 0.95 & 0.97 & 1.02 \\
\hline 7 & 2,2',3,3',4,5,6'-Heptachlorobiphenyl & PCB174 & 1.00 & 1.11 & 1.12 \\
\hline 8 & $1,2,3,4,6,7,8,9$-Octachlorodibenzofuran & $\mathrm{OCDF}$ & 1.00 & 0.97 & 1.06 \\
\hline $9 *$ & 2,3,3',4',6-Pentachlorobiphenyl & PCB110 & 1.04 & 0.96 & 1.31 \\
\hline 10 & 2,2',4,4',5-Pentachlorobiphenyl & PCB99 & 1.11 & 1.15 & 1.24 \\
\hline 11 & 2,2',4,5,5'-Pentachlorobiphenyl & PCB101 & 1.25 & 1.18 & 1.12 \\
\hline 12 & 2,3,7,8-tetrachlorodibenzo-p-dioxin & TCDD & 1.25 & 1.21 & 1.32 \\
\hline 13 & 2,3',4,4',5-Pentachlorobiphenyl & PCB118 & 1.30 & 1.09 & 1.25 \\
\hline 14 & 3,3',4,4',5,5'-Hexachlorobiphenyl & PCB169 & 1.32 & 1.41 & 1.48 \\
\hline $15^{*}$ & 2,3,3',4,4'-Pentachlorobiphenyl & PCB105 & 1.36 & 1.14 & 1.20 \\
\hline $16^{*}$ & 2,2',3,3',4,4',6-Heptachlorobiphenyl & PCB171 & 1.36 & 0.85 & 1.44 \\
\hline 17 & 2,2',3,4,5,5'-Hexachlorobiphenyl & PCB141 & 1.43 & 1.30 & 1.39 \\
\hline 18 & 2,2',3,4,4',5',6-Heptachlorobiphenyl & PCB183 & 1.43 & 1.52 & 1.30 \\
\hline 19 & 2,2',3,3',4,4',5,5'-Octachlorobiphenyl & PCB194 & 1.43 & 1.55 & 1.62 \\
\hline $20 *$ & 2,2',3,4,4',5,5',6-Octachlorobiphenyl & PCB203 & 1.43 & 1.46 & 1.47 \\
\hline 21 & 3,3',4,4',5-Pentachlorobiphenyl & PCB126 & 1.43 & 1.31 & 1.23 \\
\hline 22 & 2,2',3,4,4',5'-Hexachlorobiphenyl & PCB138 & 1.46 & 1.34 & 1.41 \\
\hline $23 *$ & 2,2',4,4',5,5'-Hexachlorobiphenyl & PCB153 & 1.46 & 1.28 & 1.25 \\
\hline 24 & 2,2',3,4',5,5'-Hexachlorobiphenyl & PCB146 & 1.48 & 1.53 & 1.41 \\
\hline 25 & 2,2',3,3',4,5',6,6'-Octachlorobiphenyl & PCB201 & 1.48 & 1.44 & 1.45 \\
\hline 26 & 2,2',3,3',4,5,6,6'-Octachlorobiphenyl & PCB200 & 1.50 & 1.49 & 1.41 \\
\hline 27 & 2,2',3,3',4,5,5'-Heptachlorobiphenyl & PCB172 & 1.53 & 1.40 & 1.57 \\
\hline $28 *$ & 2,2',3,4,4',5,5'-Heptachlorobiphenyl & PCB180 & 1.53 & 1.45 & 1.48 \\
\hline 29 & 1,1-Dichloro-2,2-(4-ClC6H4)ethane & $\mathrm{p}, \mathrm{p}$ 'DDD & 1.61 & 1.81 & 1.67 \\
\hline $30 *$ & Dichlorodiphenyltrichloroethane & DDT & 1.92 & 2.02 & 2.37 \\
\hline 31 & 1,1-Dichloro-2,2-(4-ClC6H4)ethene & $\mathrm{p}, \mathrm{p}^{\prime}-\mathrm{DDE}$ & 2.19 & 1.96 & 2.14 \\
\hline $32 *$ & 1,2,3,6,7,8-Hexachlorodibenzo-p-Dioxin & H6CDD & 2.44 & 2.15 & 2.11 \\
\hline 33 & 1,2,3,4,6,7,8-Heptachlorodibenzo-p-Dioxin & $\mathrm{H} 7 \mathrm{CDD}$ & 2.44 & 2.42 & 2.33 \\
\hline 34 & $\begin{array}{l}\text { 1,2,3,4,6,7,8,9-Octachlorodibenzo-p- } \\
\text { Dioxin }\end{array}$ & OCDD & 2.49 & 2.58 & 2.48 \\
\hline 35 & $2,3^{\prime}, 4,4^{\prime}$-Tetrachlorobiphenyl & PCB66 & 0.83 & 1.04 & 0.93 \\
\hline 36 & 2,2',3,5',6-Pentachlorobiphenyl & PCB95 & 0.83 & 0.88 & 0.83 \\
\hline 37 & 2,2',3,3',4,4',5-Heptachlorobiphenyl & PCB170 & 1.53 & 1.46 & 1.59 \\
\hline 38 & 2,3,3',4,4',5,6-Heptachlorobiphenyl & PCB190 & 1.53 & 1.50 & 1.68 \\
\hline
\end{tabular}




\begin{tabular}{llllll}
39 & $2,2^{\prime}, 3,4,4^{\prime}, 5,6^{\prime}-$ Heptachlorobiphenyl & PCB182 & 1.39 & 1.47 & 1.28 \\
40 & $2,2^{\prime}, 3,4^{\prime}, 5,5^{\prime}, 6$-Heptachlorobiphenyl & PCB187 & 1.39 & 1.39 & 1.25 \\
\hline
\end{tabular}

315 
Table 2 Performance of the established models

\begin{tabular}{clcc}
\hline Data set & \multicolumn{1}{c}{ Item } & TM & 2D-QSPR \\
\hline Training & $L V_{c}$ & 5 & 6 \\
& $R_{c}$ & 0.9726 & 0.9732 \\
& $R_{a d j}$ & 0.9668 & 0.9661 \\
& $R_{c v}$ & 0.9570 & 0.9545 \\
& $R M S E_{c}$ & 0.1052 & 0.1040 \\
& $R M S E_{c v}$ & 0.1320 & 0.1353 \\
& $F-t e s t(p$-value $)$ & $2.08 \mathrm{E}-14$ & $1.56 \mathrm{E}-13$ \\
& $R_{r}{ }^{2}$ & 0.5669 & 0.3065 \\
& ${ }^{c} R_{p}{ }^{2}$ & 0.5988 & 0.7790 \\
& $R_{p}$ & 0.9594 & 0.9236 \\
& $R M S E_{p}$ & 0.2129 & 0.2541 \\
& $k$ & 1.0783 & 0.9757 \\
& $k^{\prime}$ & 0.9129 & 0.9952 \\
& $r_{m}{ }^{2}$ & 0.8567 & 0.7685 \\
& $r_{m}{ }^{2}$ & 0.9161 & 0.6220 \\
& $\Delta r_{m}{ }^{2}$ & 0.0594 & 0.1465 \\
\hline & & &
\end{tabular}

Table 3 Comparison of the predicted results for the five test sets

\begin{tabular}{cccrc}
\hline & \multicolumn{3}{c}{$R_{p}{ }^{2}$} & \multicolumn{2}{c}{$R M S E_{p}$} \\
\cline { 2 - 5 } Test set & TM & aug-MIA-QSPR color & TM & aug-MIA-QSPR color \\
& 0.9710 & 0.8451 & 0.0792 & 0.2310 \\
test 1 & 0.9877 & 0.7808 & 0.0589 & 0.2261 \\
test 2 & 0.9844 & 0.8719 & 0.0803 & 0.2048 \\
test 3 & 0.9854 & 0.8759 & 0.0590 & 0.1957 \\
test 4 & 0.9860 & 0.8978 & 0.0690 & 0.2061 \\
test 5 & $0.9829 \pm 0.0068$ & $0.8543 \pm 0.0452$ & $0.0693 \pm 0.0104$ & $0.2127 \pm 0.0151$ \\
Average \pm SD & & & & \\
\hline
\end{tabular}


Figure 1

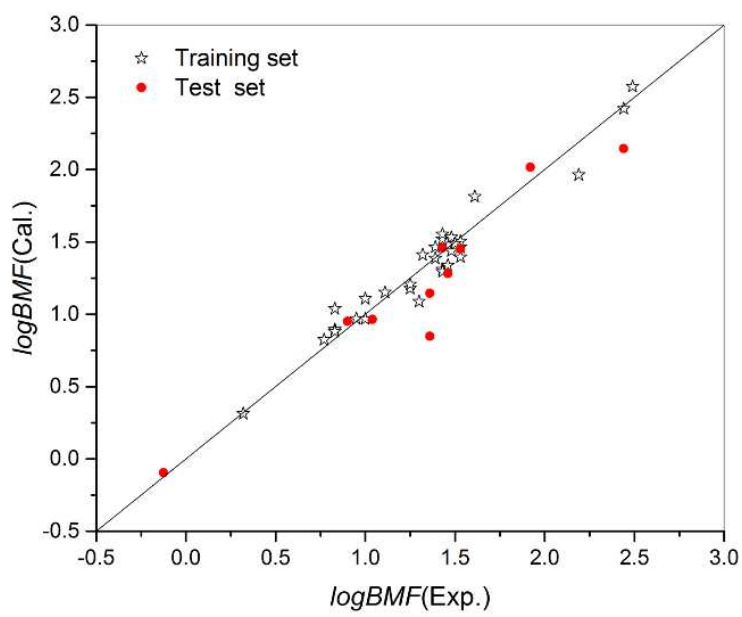

2

$3 \quad$ Figure 2
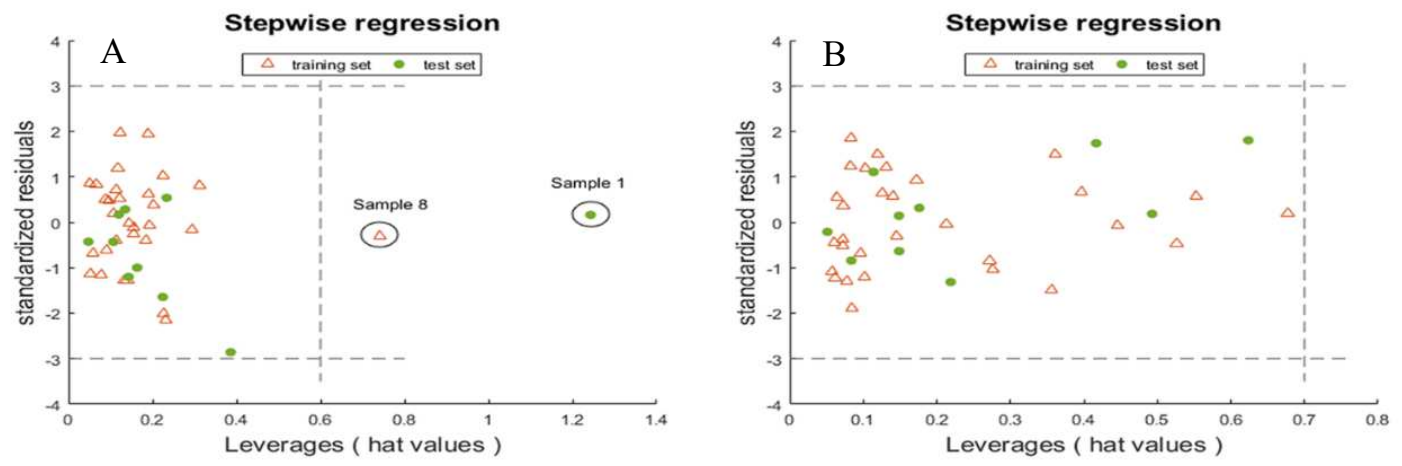
Figures

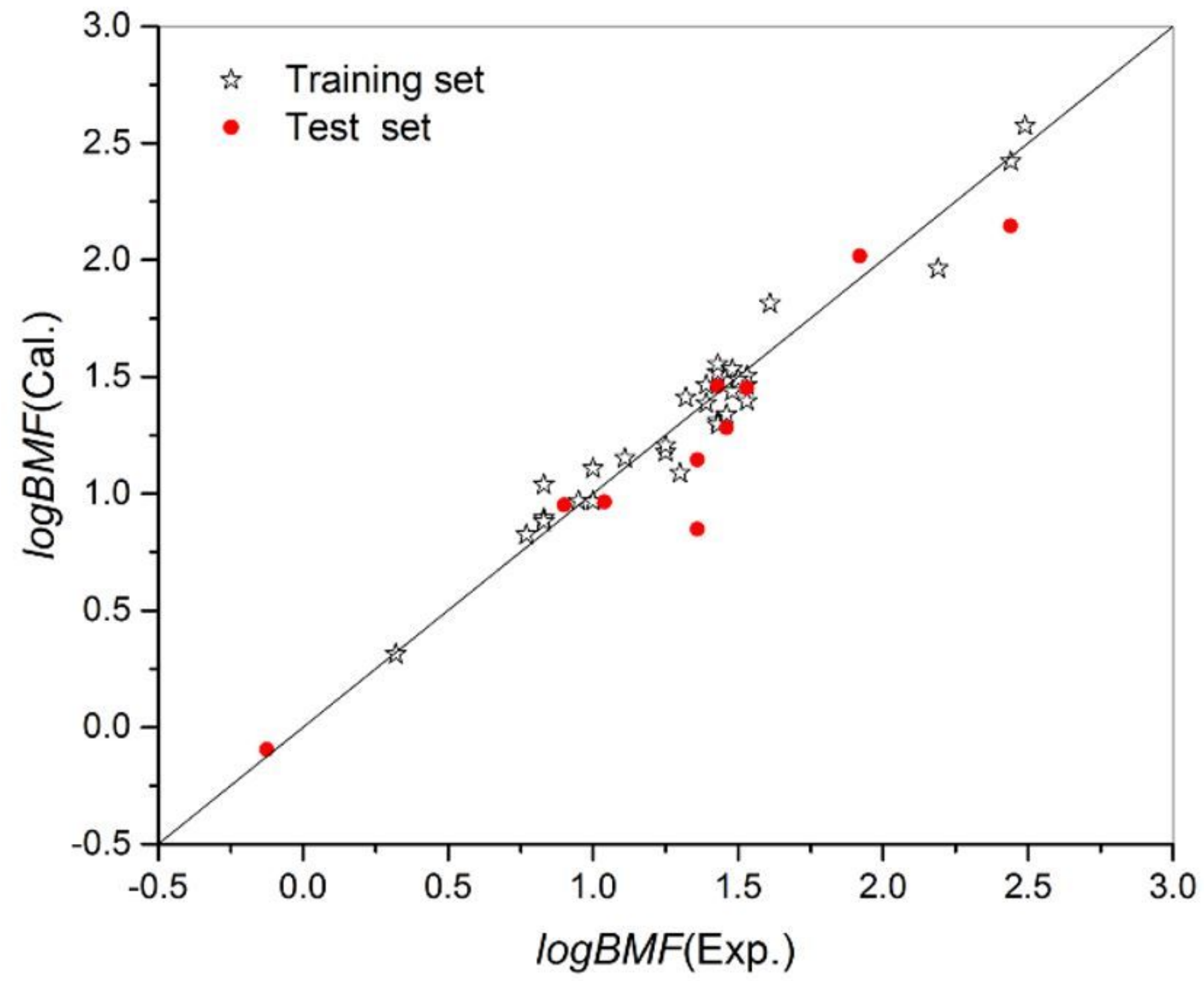

Figure 1

Linear relationship between logBMF calculated values and experimental values 

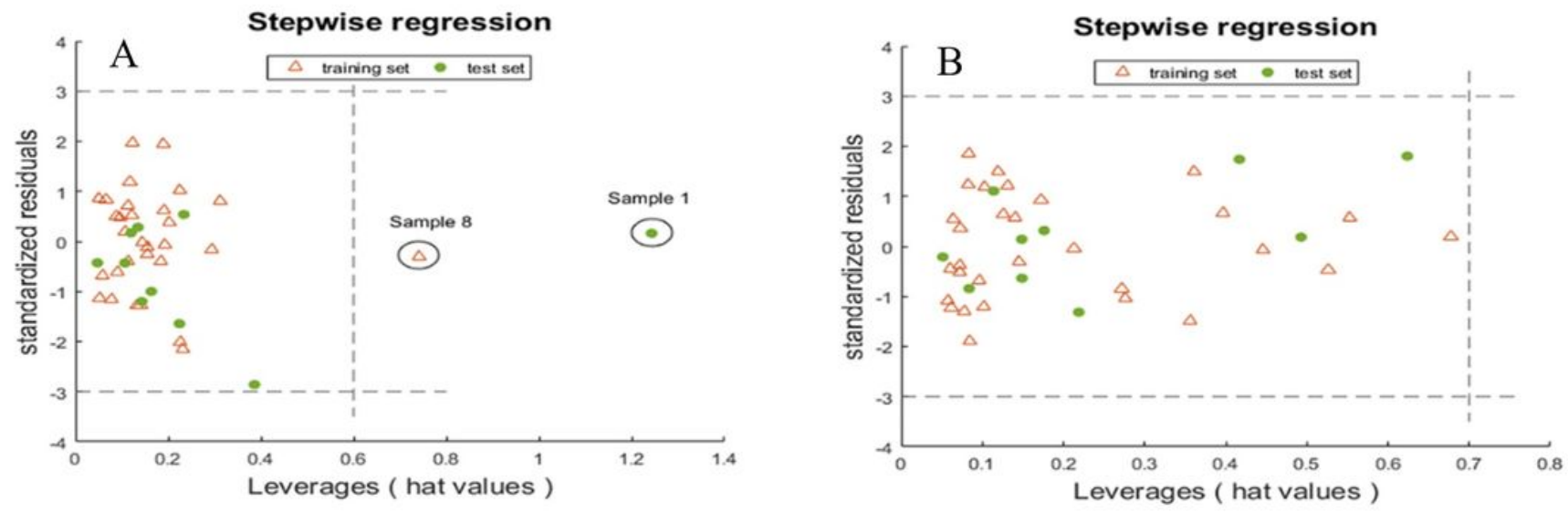

Figure 2

Williams plots. (A) TM model $\left(H^{\star}=0.6\right)(B) 2 D-Q S P R$ model $\left(H^{*}=0.7\right)$

\section{Supplementary Files}

This is a list of supplementary files associated with this preprint. Click to download.

- Supportinginformation.docx 